\title{
Una Novella Laica: I Tre Anelli
}

\author{
A Secular Novella: The Three Rings
}

(dalla I giornata del Decameron) di G. BOCCACCIO

Daniele STABELLINI*

Sommario: Nelle 101 novelle del Decameron emerge una società in un'epoca di forte trasformazione, tra mentalità medievale e un nuovo modo di pensare che è alla base dello sviluppo della società commerciale, di casa in tutto il Mediterraneo. La società dei mercanti ha messo a fuoco che le premesse per un proficuo commercio tra le diverse comunità del Mediterraneo sono l'accettazione dell"altro"e il rispetto reciproco. E' un modo nuovo di pensare di una nuova parte della società, una nuova categoria economica. I mercanti cercano e trovano un modo pragmatico di considerare le differenze tra le tre religioni monoteiste non più ostacolo né strumento di divisione e lotta. Tutto si sviluppa in ambiente medio -orientale, nel quale si mescolano i bisogni economici e le riflessioni "politiche" del Sultano, la consolidata agiatezza delle comunità ebraiche e la ragion di Stato, moderata da un senso di giustizia e di opportunità. Quali le radici di questa nuova mentalità?

Parole Chiavi: Anelli, Cristianesimo, Ebraismo, Islam, monoteismo, Mediterraneo, Federico II, Sultano

Abstract: The 101 short stories in the Decameron portray a society during an era of great transformation, between the medieval and a new perspective that underlies the development of the commercial society, at home around the Mediterranean Sea. It's a new way of thinking in a new part of society, a new economic category. Merchants find that acceptance of the "other" and mutual respect are needed in order to get a profitable trade between different communities of the Mediterranean. The merchants seek and find pragmatic ways to consider the differences between the three monotheistic religions as no longer being obstacles or instrument of division and struggle. The plot is developed in the Middle East, in which economic needs and the political considerations of the Sultan, the consolidated wealth of Jewish communities and Raison d'Etat, moderated by a sense of justice and opportunity mingle. What are the roots of this new pragmatic mentality?

Keywords: Rings, Christianity, Judaism, Islam, monotheism, Mediterranean, II. Frederick, Sultan

Il Saladino, il valore del quale fu tanto grande che il destino non solo da piccolo uomo lo fece diventare Sultano di Babilonia, ma gli fece anche avere molte vittorie sui Re saraceni e cristiani, aveva speso tutto il suo tesoro in diverse guerre e in grandissimo lusso.

Fu così che poi, per alcuni imprevisti, ebbe bisogno di una buona quantità di denari, e non sapeva dove poterli trovare così in fretta. Gli venne allora in mente un ricco ebreo, il cui nome era Melchisede, il quale prestava soldi a usura ad Alessandria. Così pensò che costui, se avesse voluto, aveva quello che gli poteva servire, ma era così avaro che di sua spontanea volontà non l'avrebbe mai dato, e il Saladino non voleva costringerlo con la forza. Fu così che, stringendo il bisogno, tutto intento a trovare come venire in possesso dei soldi del giudeo, si decise a costringerlo,

* PhD. Cand., Université Paris Ouest et, Università Alma Mater, Bologna, danstab@hotmail.com 
in un modo apparentemente ragionevole (violenza con apparenza di ragione). E fattolo chiamare, e ricevutolo familiarmente, lo fece sedere con sé, e poi gli disse:

"Valente uomo, io ho saputo da molte persone che tu sei molto saggio, $e$ sei molto esperto nelle cose di Dio; e per questo io volentieri vorrei sapere da te, quale delle tre religioni ritieni la più vera: o la giudaica, o la musulmana, o la cristiana".

L'ebreo, il quale era veramente un uomo saggio, capì fin troppo bene che il Saladino cercava di incastrarlo con le parole, per poi poterlo poi accusare, e pensò di non poter lodare alcuna di queste tre religioni più dell'altra, senza che il Saladino raggiungesse il suo scopo. Sicché, avendo bisogno di una risposta incontestabile, aguzzò l'ingegno e gli venne presto in mente quello che dovesse dire, e disse:

"Signor mio, la domanda che voi mi fate è bella, e per dire ciò che io credo, mi vien da raccontarvi una novella, che voi ascolterete. Se io non erro, io mi ricordo di aver sentito dire molte volte che c'era un uomo grande e ricco, il quale, tra gli altri gioielli più cari che aveva nel suo tesoro, possedeva un anello bellissimo e prezioso; volendogli fare onore, per il suo valore e per la sua bellezza, e lasciarlo per sempre ai suoi discendenti, ordinò che quello dei suoi figliuoli che si fosse trovato con questo anello, in quanto avutolo da lui, si considerasse il suo erede, $e$ dovesse da tutti gli altri essere onorato e riverito come tale.

Colui al quale l'anello fu lasciato mantenne la stessa disposizione verso $i$ suoi discendenti, e cosi fece il suo erede: e, per dirla in breve, questo anello andò di mano in mano a molti successori; e ultimamente pervenne in mano ad uno, che aveva tre figliuoli belli e virtuosi, e molto obbedienti al padre; per la qual cosa egli amava tutti e tre nello stesso modo. E $i$ giovani, che conoscevano la consuetudine dell'anello, pregavano il padre, che era già vecchio, come meglio sapevano, ciascuno per sé affinché, quando dovesse morire, lasciasse a lui quell'anello, come se ognuno sognasse d'essere il più onorato tra tutti, Il valente uomo, che li amava tutti allo stesso modo, non sapeva decidere a quale figlio volesse lasciare l'anello; e avendolo promesso ad ognuno, pensò di volerli soddisfare tutti e tre; e segretamente ne fece fare altri due da un buon maestro, cosi uguali al primo, che lui stesso che li aveva fatti, a mala pena riconosceva quale fosse quello vero.

E poco prima di morire, segretamente diede un anello a ciascuno dei figli; dopo la morte del padre, ciascun fratello voleva ottenere l'eredità e l'onore, che reciprocamente ciascuno negava all'altro; allora, come prova di giusta pretesa, ognuno tirò fuori il suo anello. E trovarono gli anelli cosi simili l'uno all'altro, che non si sapeva riconoscere quale fosse il vero. Rimase cosi irrisolta la domanda: chi era il vero erede del padre? Questa domanda è ancora oggi senza risposta.

E così vi dico, signor mio, delle tre religioni date da Dio Padre ai tre popoli, di cui avete proposto il dilemma: ciascuno crede di avere la sua eredità, la sua vera Legge, e i suoi comandamenti; ma chi abbia quella vera, come degli anelli, ancora non si può sapere".

Il Saladino riconobbe che l'ebreo aveva saputo uscire ottimamente dalla trappola che gli aveva teso davanti ai piedi: e per questo si decise a confidargli la sua necessità, e vedere se volesse 


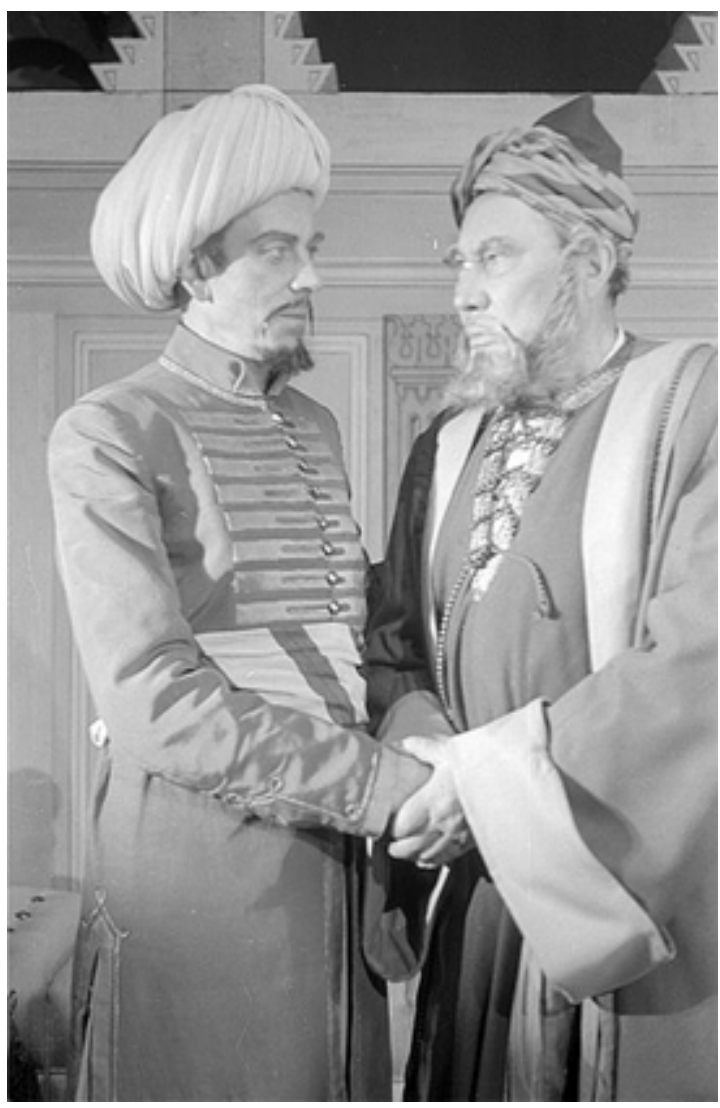

aiutarlo; e così fece, rivelandogli ciò che aveva avuto in animo di fare, se non gli avesse risposto così amabilmente, come invece aveva fatto.

Il giudeo lo aiutò volentieri d'ogni quantità che il Saladino richiese; e il Saladino poi lo rimborsò interamente; e oltre a ciò gli fece grandissimi doni, e l'ebbe sempre per suo amico e lo tenne con sé in grande considerazione (Versione dall'originale (C) Daniele Stabellini, $31 / 3 / 2012$ ).

Ho voluto iniziare con questa bella novella di Giovanni Boccaccio una riflessione su alcune delle caratteristiche fondamentali dell'opera letteraria che Boccaccio ci ha lasciato. Nelle 101 novelle del Decameron, infatti, emerge una società in un'epoca di forte trasformazione, tra ciò che resta della mentalità medievale e un nuovo modo di pensare che è alla base dello sviluppo della società commerciale, ormai di casa in tutto il Mediterraneo. Questa nuova maniera di interpretare il mondo mostra come nel Trecento il dibattito su ciò che oggi chiamiamo laicità e tolleranza fosse già maturo e ben strutturato. La società dei mercanti aveva già messo ben a fuoco che le premesse per un proficuo commercio tra le diverse comunità che solcavano il Mediterraneo sono l'accettazione dell"'altro"e il rispetto reciproco. E' in effetti un modo nuovo di pensare di una nuova parte della società, una nuova categoria economica che si confronta, pur nelle differenze religiose che ancora le caratterizzano, per valorizzare ciò che le unisce, senza esasperare ciò che divide. Novelle come questa, che girano nei porti e nelle maggiori città commerciali dei paesi che si affacciano sul Mediterraneo cominciano a tessere una nuova realtà culturale ed ideologica, pongono le basi per unire su un terreno comune -il commercio- popoli fino a qualche tempo prima, e per molto tempo ancora, divisi da ideologie e credi contrapposti. Sono proprio i mercanti che cercano e trovano un modo nuovo, molto pragmatico e poco ideologico, di considerare le differenze tra le tre religioni monoteiste non più un ostacolo, ma anzi un elemento positivo, tre bei doni sostanzialmente simili e di eguale natura, frutto dell'amore di un Padre per i figli, non certo strumento di divisione e lotta.

Il testo, che Boccaccio ci tramanda, notiamo subito che si sviluppa in un ambiente medio orientale, nel quale si mescolano i bisogni economici e le riflessioni "politiche" del Sultano, la consolidata agiatezza delle comunità ebraiche, la ragion di Stato moderata da un senso di giustizia e opportunità che pur caratterizzò il governo di un potere che non esitò a espandersi sopra ogni altro.

Ma non è nei riferimenti storici pur rilevanti che si trova l'importanza della novella. Accanto all' eleganza di un Sultano che non ama far violenza ai suoi sudditi, caratteristica narrativa che riporta in occidente una certa visione di un oriente idealizzato, in poche righe vengono a mio avviso ridimensionate e relativizzate le idee più tipiche dei monoteismi in questione: l'unicità e l'originalità. Il valore intrinseco dell'anello si basa infatti sulla sua caratteristica di essere unico e questa è la garanzia dell'eredità del Padre. Evidentemente le "copie" indistinguibili introducono 
un elemento di relatività, ovvero la sostanziale assenza di primato di un anello sugli altri.

Anzi, ciò che prima era percepita come un'inguaribile diversità e quindi inconciliabile opposizione, diventa nella novella un vero e proprio messaggio d'amore, che invita i fratelli a governare insieme il patrimonio del Padre, senza divisioni né contrasti, in un'armonia che mette le tre religioni su uno stesso piano, senza che nessuna possa rivendicare alcun primato, così come indistinguibili sono i tre anelli. C'è insomma la nuova idea della sostanziale somiglianza e quindi dell'intercambiabilità delle tre religioni, come tre modi diversi ma non antitetici di vivere nel rispetto del volere del Padre e del suo identico amore per tutti i suoi figli.

Su questo valore di una tolleranza che sfocia nella laicità, presentate così come genuinamente naturali, si basa un nuovo patrimonio culturale di una società che ha scoperto come superare il vicolo cieco dell'integralismo, che sperimenta ogni giorno in ogni mercato che solo su questi valori possono svilupparsi, attraverso gli scambi, i traffici e i commerci, nuovi rapporti per una nuova convivenza tra $\mathrm{i}$ popoli.

Il primato di ciò che è ragionevole e giusto (e quindi della laicità) emerge anche dalla decisione finale del Sultano, che rinuncia alla prevaricazione e, di buon accordo con l'ebreo, mostra un modello di governo che oggi chiameremmo "sostenibile", basato sull'interesse reciproco di chi è al potere e chi, pur senza ancora alcun contratto sociale né garanzie di diritti, trova nella saggezza di chi governa un elemento di equilibrio.

Ecco infatti che la novella ci mostra le aspettative di quella classe mercantile così attenta anche alla tolleranza religiosa: un modello di governo che saggiamente sappia rinunciare non solo alla prevaricazione, alla forza, all'estorsione, ma persino a quella violenza con apparenza di ragione che potrebbe sembrare giustificabile. E' un'aspettativa senza mezzi termini, un ideale di rispetto completo di chi governa verso chi è governato, per tutti, sudditi fedeli ed infedeli. C'è un alto senso dello Stato, di un potere super partes, che presta attenzione e rispetto perfino alla parte meno garantita della società, quella che oggi definiremmo la minoranza: avere un occhio di riguardo per i soli propri fedeli, i quali per il solo fatto di esser tali già si presuppone siano trattati meglio degli infedeli, non sarebbe stata la stessa cosa.

La mente corre ad un'idea moderna, che prenderà forma esplicita solo qualche secolo dopo, quella del potere che rispetta il suo popolo, tutto, senza distinzioni di religione, come in questo caso. Ma anche a un'altra idea ancor più moderna: il supporto economico delle classi abbienti all'economia del proprio Paese. Se con la prima idea siamo già fuori dall'orizzonte medievale che vedeva il rapporto tra potenti e sudditi come essenzialmente un rapporto basato sull'arbitrio e la violenza, anche la seconda idea ci mostra una nuova visione delle relazioni tra governati e governanti, fatta di rispetto reciproco, di collaborazione, condivisione, perfino amicizia. C'è un abisso tra la realtà storica del XIV secolo e le aspettative della classe mercantile, che già profetizza un'utopia che si metterà a fuoco solo dopo l'Illuminismo.

Ma in questa novella c'è più ragione che passione, c'è la volontà di raccontare che un nuovo modello sociale è possibile, anzi, è inevitabile. La passione avrebbe portato ad usare la forza, scatenando probabilmente una reazione ostile da parte della comunità vessata, alla quale sarebbe stato difficile non contrapporre con altrettanta ostilità chi già mal sopportava le ricchezze e gli agi dei ricchi mercanti, ebrei o non ebrei. Ma questa sarebbe stata cronaca, storia, non letteratura.

Come scriveva Dante:

..."I'mi son un che, quando

Amor mi spira, noto, e a quel modo

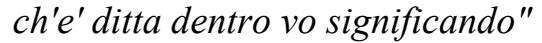

("Donne ch'avete intelletto d'amore", in Divina Commedia, Purgatorio, Canto XXIV). 
Anche Boccaccio conferma, pur se indirettamente, che compito della letteratura, non solo della poesia ma anche della prosa, è prender nota di ciò che è ragionevole (e in quanto tale degno di essere amato) ovvero ciò che è ispirato dall'amore, l'amore nel senso più alto di intelletto, ovvero ragione, in questo caso. Ma non si limita ad annotare, invece intende proprio dire in modo esplicito, dar significato a ciò che la ragione va dettandoci dentro, va facendoci capire. Ecco il tesoro che ci lascia la letteratura: poter cogliere i primi fermenti di qualcosa che cambia, come un dolce venticello che ci porta fuori dalla mente ciò che par ragionevole, cioè che ci fa render conto di ciò che è preferibile, spesso in contrasto con la realtà. Questo "pre-sentimento" è spesso precursore di cambiamenti ineluttabili, che si consolideranno non appena questo venticello avrà preso forza e coraggio e avrà diffuso questo nuovo modo di intendere la realtà.

Il rifiuto della violenza, della passionalità, dell'irrazionalità, porta dunque ad un diverso rapporto tra il potere e il popolo, un rispetto aperto anche del "diverso", all'altro; questo di conseguenza apre ad una nuova relazione tra il suddito e il potere, fatto di disponibilità e di supporto, di sostegno ed appoggio in virtù della garanzia non scritta, ma non per questo meno sicura, che il prestito verrà rimborsato interamente, portandosi dietro anche un bonus di un sovrappiù di grandissimi doni, amicizia e maggior considerazione.

C'è la visione di una società nuova, fatta di solidarietà tra le diverse componenti, articolata su reciproco vantaggio, mutua convenienza, su sentimenti positivi come l'amicizia e la stima. Ma i mercanti la sapevano lunga, avevano già sperimentato da un lato l'insufficienza e l'inefficienza dell'angusta società feudale e dall'altro la possibilità di generalizzare quanto nel loro piccolo venivano sperimentando come fattore di prosperità e successo: la tolleranza, il rispetto reciproco, l'abbandono della violenza in favore della fiducia reciproca, della negoziazione, della diversa ma complementare funzione delle varie parti sociali. Sono i mercanti, in primis, che abbandonata la teoria dello scontro ben volentieri si incontrano, musulmani, cristiani, ebrei, nei mercati del Mediterraneo e si rendono conto, insieme, senza nessuna teorizzazione necessaria, che è possibile commerciare, scambiarsi merci e idee, esperienze e leggende, aver tutti a trarre profitti maggiori da quel nuovo clima culturale, di cui si era già respirata una sana ventata nel centro del Mediterraneo alla corte di Federico II di Svevia a Palermo, dove era fiorito tra il 1230 e il 1250 un forte e nuovo movimento culturale, frutto saporito di uno stato operoso, ordinato e pacifico.

L'imperatore che fu, secondo papa Gregorio IX:

"...la bestia che sorge dal mare carica di nomi blasfemi, e infuriando con la zampa dell'orso e le fauci del leone, informata nelle restanti membra a guisa di leopardo spalanca la bocca ad offesa del Santo Nome senza cessare di scagliare la stessa lancia sul tabernacolo di Dio e Sui Santi che abitano nei cieli..."

(http://www.stupormundi.it/Federico-II.htm, 04/05/12).

o invece

"il salvatore inviato da Dio, il principe della pace, il messia-imperatore"

come lo definì Pier delle Vigne (Dante Alighieri, Divina Commedia, Inferno, XIII), dapprima notaio di corte, poi giudice ed infine protonotaro (ministro), da sempre suo amico e confidente, passò alla storia come lo Stupor Mundi, un uomo di ingegno eccezionale, universalmente considerato superiore ad ogni altro personaggio contemporaneo. Fu letterato, statista, condottiero, legislatore; ...

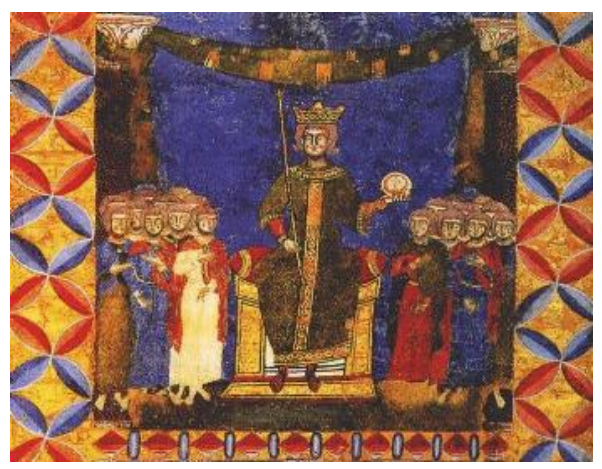


è ricordato soprattutto per la mentalità libera, eclettica ed anticipatrice (...) che seppe interpretare contemporaneamente molteplici situazioni che oggi ci appaiono fra loro apertamente contrastanti. E ciò non per incoerenza o scaltro opportunismo, ma perché dovette dominare un periodo di profondo cambiamento, dibattuto fra integralismo ... e stato laico; superstizione e scienza nascente; dogmatismo, eresie e libero pensiero ... in ogni situazione egli fornì sempre un esempio di libertà interpretativa e di tolleranza ideologica difficilmente inquadrabile in una religione $\mathrm{o}$, più in generale, in un movimento di pensiero organizzato (...) egli era già pervenuto al concetto dell'unità e della universalità del sapere umano, per cui veniva abolita ogni differenza fra un dotto cristiano, musulmano ed ebreo ... Senza discriminazione di razza e di fede, la sua Corte accolse tutti i principali uomini di cultura che in quel momento erano portatori delle teorie di maggiore avanguardia, attingendo soprattutto dalle più avanzate scuole d'Oriente (http://www.stupormundi.it/lacorte.htm, 05/05/12).

In una mirabile fusione di culture: greci, normanni, arabi, latini tutti insieme contribuirono a fare di Palermo la più avanzata esperienza culturale dell'epoca. E questo fatto gettò i presupposti per un nuovo clima nel Mediterraneo che dapprima solo i mercanti seppero cogliere e far proprio, passando dalle aspirazioni ideali alla pratica attuazione, nella quotidiana esperienza, e diffon-dendolo pian piano in tutti porti del Mediterraneo, come racconto, come sogno, come ideale o come realtà quotidiana, preconizzando così un nuovo modo di intendere gli scambi, non di soli beni ma soprattutto di quei germi di rinnovamento sociale che prima o poi dovevano sbocciare, fiorire e dare frutti.

Nella novella, insomma, e soprattutto nelle sue conclusioni, c'è abbastanza, per riflettere e far riflettere sulla saggezza di ieri e sull'insipienza di oggi.

\section{Il testo originale: I TRE ANELLI. (Giovanni Boccaccio, Decameron, I,3)}

Il Saladino, il valore del qual fu tanto che non solamente di piccolo uomo il fe' di Babilonia Soldano, ma ancora molte vittorie sopra li Re saracini e cristiani li fece avere, avendo in diverse guerre, et in grandissime sue magnificenze, spese tutto il suo tesoro, e, per alcuno accidente sopravvenutogli, bisognandogli una buona quantità di danari, né veggendo donde così prestamente, come gli bisognavano, aver li potesse, gli venne a memoria un ricco giudeo, il cui nome era Melchisedech, il quale prestava ad usura in Alessandria, e pensossi costui avere da poterlo servire quando volesse; ma si era avaro che di sua volontà non l'avrebbe mai fatto, e forza non gli voleva fare: per che, stringendolo il bisogno, rivoltosi tutto a dover trovar modo come il giudeo il servisse, s'avvisò di fargli una forza da alcuna ragion colorata (violenza con apparenza di ragione).

E fattolsi chiamare, e familiarmente ricevutolo, seco il fece sedere, et appresso gli disse: Valente uomo, io ho da più persona inteso che tu se' savissimo, e nelle cose di Dio senti molto avanti; e per ciò io saprei volentieri da te, quale delle tre Leggi tu reputi la verace, o la giudaica, o la saracina, o la cristiana. Il giudeo, il quale veramente era savio uomo, s'avvisò troppo bene che il Saladino guardava di pigliarlo nelle parole, per dovergli muovere alcuna quistione, e pensò non potere alcuna di queste tre più l'una che l'altra lodare, che il Saladino non avesse la sua intenzione. Per che, come colui il qual parea aver bisogno di risposta per la quale preso non potesse essere, aguzzato lo 'ngegno, gli venne prestamente avanti quello che dir dovesse, e disse:

"Signor mio, la quistione la qual voi mi fate è bella, et a volerne dire ciò che io ne sento, mi vi convien dire una novelletta, qual voi udirete. Se io non erro, io mi ricordo aver molte volte udito dire che un grande uomo e ricco fu già, il quale, intra l'altre gioie più care che nel suo tesoro avesse, era uno anello bellissimo e prezioso; al quale per lo suo valore e 
per la sua bellezza volendo fare onore, et in perpetuo lasciarlo ne' suoi discendenti, ordinò che colui dei suoi figliuoli appo il quale, sì come lasciatogli da lui fosse questo anello trovato, che colui s'intendesse essere il suo erede, e dovesse da tutti gli altri essere, come maggiore, onorato e reverito.

Colui al quale da costui fu lasciato tenne somigliante ordine ne' suoi discendenti, e così fece come fatto avea il suo predecessore: et in breve andò questo anello di mano in mano a molti successori; et ultimamente pervenne alle mani ad uno, il quale avea tre figliuoli belli e virtuosi, e molto al padre loro obbedienti; per la qual cosa tutti e tre parimente gli amava. Et $i$ giovani, li quali la consuetudine dell'anello sapevano, si come vaghi ciascuno d'essere il più onorato tra i suoi, ciascuno per sé, come meglio sapeva, pregava il padre, il quale era già vecchio, che, quando a morte venisse, a lui quello anello lasciasse.

Il valente uomo, che parimente tutti gli amava, né sapeva esso medesimo eleggere a qual più tosto lasciar lo volesse, pensò, avendolo a ciascuno promesso, di volergli tutti e tre soddisfare; e segretamente ad uno buono maestro ne fece fare due altri, li quali si furono somiglianti al primiero, che esso medesimo che fatti gli aveva fare, appena conosceva qual si fosse il vero.

E venendo a morte, segretamente diede a ciascuno de' figliuoli, li quali, dopo la morte del padre, volendo ciascuno la eredità e l'onore occupare, e l'uno negandolo all'altro, la testimonianza di dover ciò ragionevolmente fare, ciascuno produsse fuori il suo anello. E trovatisi gli anelli si simili l'uno all'altro, che qual fosse il vero non si sapeva conoscere, si rimase la quistione, qual fosse il vero erede del padre, in pendente, et ancor pende.

E così vi dico, signor mio, delle tre Leggi alli tre popoli date da Dio Padre, delle quali la quistion proponeste: ciascuno la sua eredità, la sua vera Legge, et i suoi comandamenti si crede avere a fare; ma chi se l'abbia, come degli anelli, ancora ne pende la quistione."

Il Saladino conobbe, costui ottimamente essere saputo uscire dal laccio il quale davanti a' piedi teso gli aveva: e per ciò dispose d'aprirgli il suo bisogno, e vedere se servire il volesse; e così fece, aprendogli ciò che in animo avesse avuto di fare, se così discretamente, come fatto avea, non gli avesse risposto.

Il giudeo liberamente d'ogni quantità che il Saladino richiese il servì; et il Saladino poi interamente il soddisfece; et oltre a ciò gli donò grandissimi doni, e sempre per suo amico l'ebbe, et in grande et onorevole stato appresso di sé il mantenne.

\section{FONTI}

Alighieri, D. (1966-67). Dante Allighieri, La Divina Commedia. Cura di G. Petrocchi, in La Comedia Secondo l'antica vulgata, Milano, Montadori Edizione.

Boccacio, G. (1980). Giovanni Boccacio, Decameron. Cura di V. Branca, Milano 1980, Einaudi. http://www.stupormundi.it/Federico-II.htm, 04/05/12

http://www.stupormundi.it/lacorte.htm, 05/05/12 